\title{
Improvement of isolation between transmitting and receiving antennas of E-plane arranged waveguide slot arrays by using layers of slit pairs with half-wavelength spacing
}

\author{
Kohei Jitosho $^{1, \text { a) }}$, Takashi Tomura ${ }^{1, \text { b) }}$, and Jiro Hirokawa ${ }^{1, c)}$ \\ ${ }^{1}$ Department of Electrical and Electronic Engineering, Tokyo Institute of \\ Technology, 2-12-1 Ookayama, Meguro, Tokyo 152-8550, Japan \\ a) jitousho.k@antenna.ee.titech.ac.jp \\ b) tomura@ee.e.titech.ac.jp \\ c) jiro@ee.e.titech.ac.jp
}

\begin{abstract}
In this paper, we propose loading layers of slit pairs with halfwavelength spacing over a corporate-feed waveguide slot array antenna in the $30 \mathrm{GHz}$ band. This structure is expected to improve the isolation between transmitting and receiving antennas arranged in the E-plane. As a result of the design, the reflection of the radiating element is suppressed under -14 $\mathrm{dB}$ over a target band $(27.8 \mathrm{GHz}-31.4 \mathrm{GHz})$. The isolation between two antennas using a $2 \times 32$-element model is improved by about $20 \mathrm{~dB}$ compared to the conventional structure without the slit-pair layers. We demonstrate this result by measurement using $64 \times 32$-element arrays.
\end{abstract}

Keywords: waveguide slot array antenna, slit-pair layer, millimeter-wave band, isolation, FWA, full-duplex

Classification: Antennas and Propagation

\section{References}

[1] Y. Miura, J. Hirokawa, M. Ando, Y. Shibuya, and G. Yoshida, "Double-layer full-corporate-feed hollow-waveguide slot array antenna in the 60-GHz-band," IEEE Trans. Antennas Propag., vol. 59, no. 8, pp. 2844-2851, Aug. 2011. DOI: 10.1109/tap.2011.2158784

[2] Y. Tsunemitsu, J. Hirokawa, M. Ando, Y. Miura, Y. Kazama, and N. Goto, "Polarization isolation characteristics between two center-feed single layer waveguide arrays arranged side-by-side," ACES J., vol. 21, no. 3, pp. 240-247, Nov. 2006.

[3] H. Arakawa, H. Irie, T. Tomura, and J. Hirokawa, "Suppression of Eplane sidelobes using double slit-layers in a corporate-feed waveguide slot array antenna consisting of $2 \times 2$-element radiating units," IEEE Trans. Antennas Propag., vol. 67, no. 6, pp. 3743-3751, June 2019. DOI: 10.1109/tap.2019.2902677 


\section{Introduction}

The double-layer corporate-feed waveguide slot array antenna was proposed for a wideband high-efficiency planar antenna in the millimeter-wave band [1]. This antenna can be used in the fixed wireless access system for simultaneous communication between transmission and reception using the same frequency. In this system, high isolation between the Tx and the Rx antennas is required. One way was based on polarization division duplex using high discrimination ability in terms of cross-polarization in the slot array antenna [2]. This system could realize about 80 $\mathrm{dB}$ isolation. Another way is based on space division multiplexing. The isolation between E-plane arranged antennas becomes worse than that of H-plane arranged antennas, affecting the radiation pattern of the slots.

We propose loading slit-pair layers over the conventional antenna. The slit spacing in the pair is set to be a half wavelength in free space. The isolation between the E-plane arranged antennas can be improved by suppressing the end-fire radiation from the slit pairs due to the propagation phase difference in the pair.

The reflection bandwidth of the radiating element is narrow for a single layer of the slit pairs. Another layer of the slit pairs is added to improve the reflection bandwidth. Dielectric layers are installed between them for the stability of the structure. The relative permittivity is 1.1. All simulations in this paper are done by HFSS.

\section{Proposed antenna}

\subsection{Structure}

Fig. 1(a) shows the structure of the proposed antenna. The conventional antenna has a double-layer waveguide structure. The bottom layer is a corporate-feed circuit and the top layer is the radiating part. A coupling slot at each end of the corporate-feed circuit excites $2 \times 2$ slots in equal amplitude as shown in Fig. 1(b). Two slit-pair layers are loaded over the conventional antenna. The slits are parallel to the H-plane and the slit spacing in the pair is designed to be a half-wavelength at the center frequency to suppress the end-fire radiation. A slit pair is placed per row of radiating slots. The upper slit-pair layer contributes to the suppression of the reflection of the radiating element [3].

\subsection{Results of design}

We design the $2 \times 2$-slot subarray with the two slit-pair layers. The center frequency is $29.6 \mathrm{GHz}$, and the target band is from $27.8 \mathrm{GHz}$ to $31.4 \mathrm{GHz}$. Two sets of periodic boundary walls are introduced in the outer region to include the mutual coupling in the infinite two-dimensional array. Fig. 1(c) shows the frequency characteristic of the reflection. When the thickness of the lower dielectric layer (\#1) is $3.1 \mathrm{~mm}$ and the thickness of the upper dielectric layer (\#2) is $1.3 \mathrm{~mm}$, the reflection is suppressed below $-14 \mathrm{~dB}$ in the target band.

\subsection{Effect of the proposed structure}

To discuss the radiation in the E-plane in simulations, we introduce an array for the Tx and Rx antennas where the number of elements in the E-plane is finite but that in 


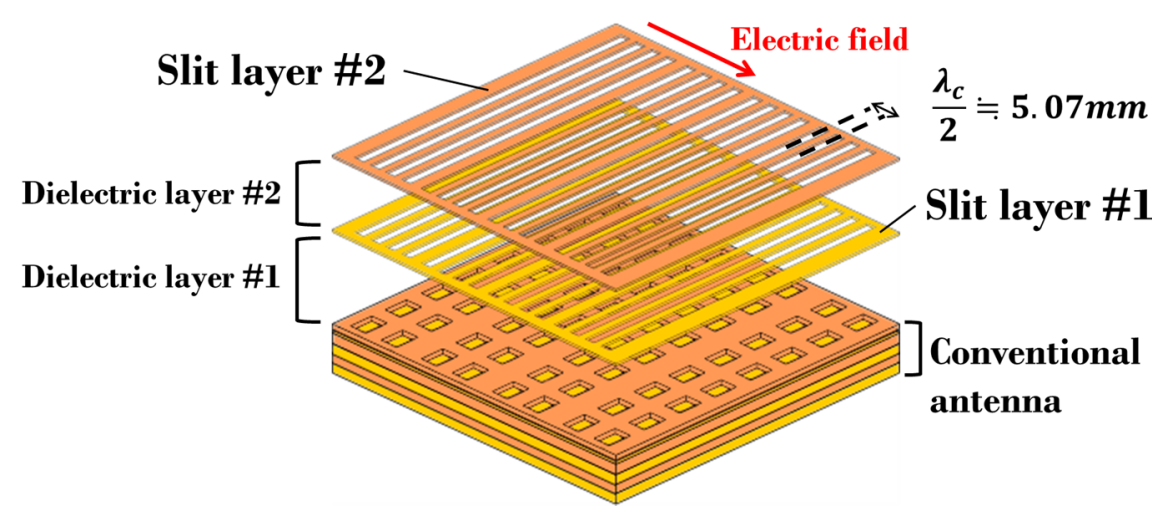

(a) Proposed antenna

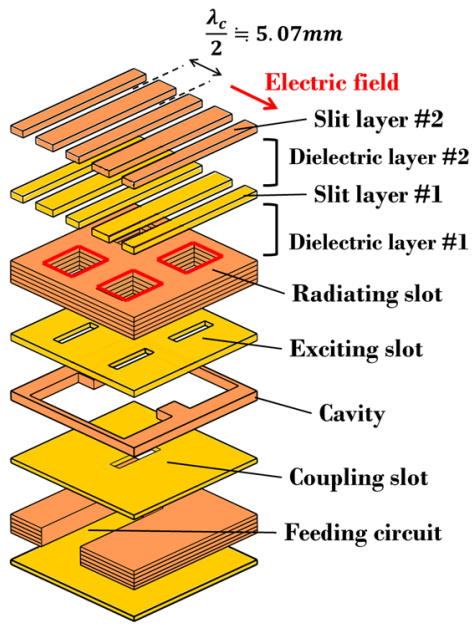

(b) 2x2-slot subarray

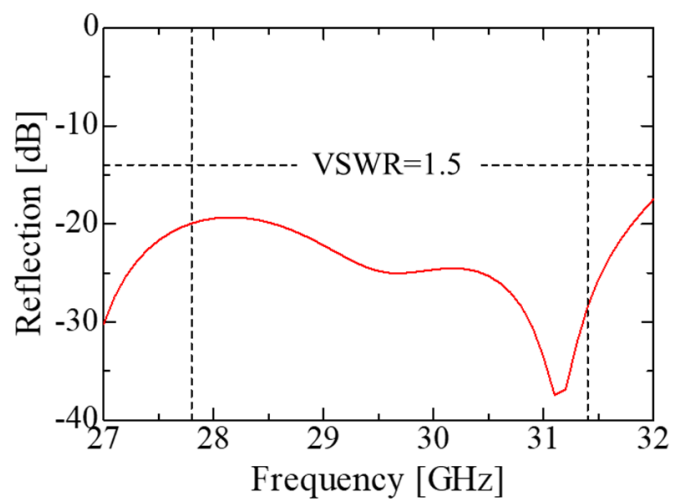

(c) Reflection in the $2 \times 2$-slot

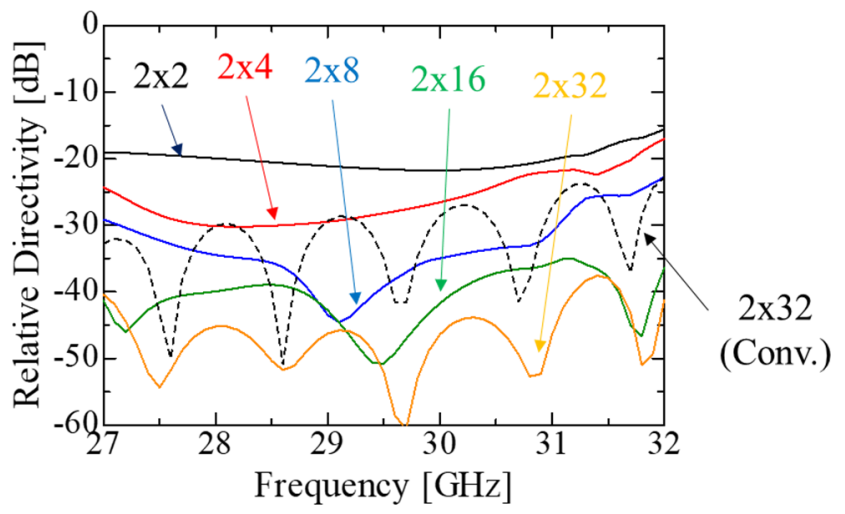

(d) Relative amplitude of the end-fire radiation

Fig. 1. Antenna structure and the results of design

the H-plane is two with periodic boundaries. Fig. 1(d) shows the relative amplitude in the end-fire direction over that in the broadside direction for various numbers of the elements in the E-plane. The relative amplitude decreases for a larger number of the elements. The proposed structure can suppress the end-fire radiation by about $20 \mathrm{~dB}$ compared to the conventional structure for a $2 \times 32$-element array.

\section{Simulation of isolation between $2 \times 32$-element arrays}


antennas arranged in the E-plane. We introduce a PML (perfect matching layer) as the radiation boundary along the E-plane and periodic boundaries along the $\mathrm{H}$-plane. The distance between the Tx and $\mathrm{Rx}$ antennas is fixed to be $10 \mathrm{~mm}\left(\approx 1.0 \lambda_{c}: \lambda_{c}\right.$ is the wavelength at the center frequency). The PML boundary is separated by $2.0 \lambda c$ from the top slit-pair layer. The results of the simulation are shown in Fig. 2(b)-(e). Each graph shows the frequency dependence of isolation.

\subsection{Improvement by the proposed structure}

Fig. 2(b) compares the isolation between two antennas with the proposed structure (w/ slit) to that between two antennas with the conventional structure (Conv.). The proposed structure can improve the isolation by about $20 \mathrm{~dB}$ compared to the conventional structure.

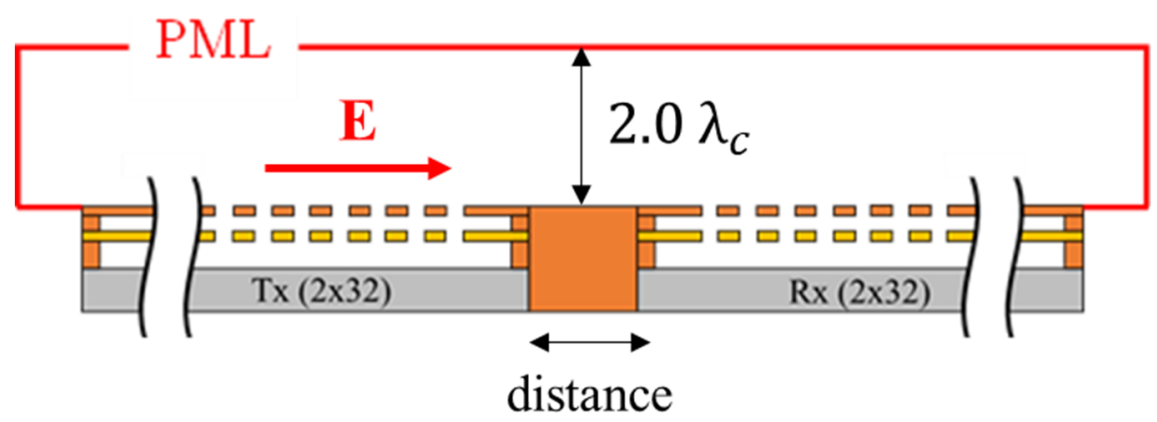

(a) Analysis model of isolation

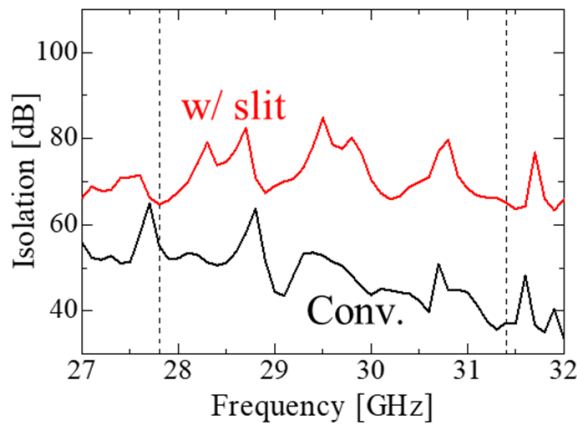

(b) Improvement by slit-pair layer

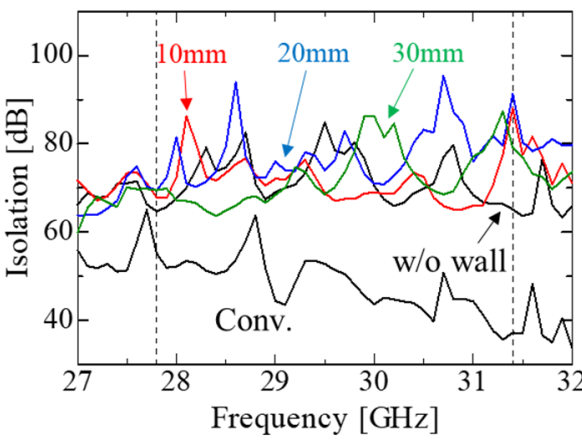

(d) Improvement by slit-pair layer and wall

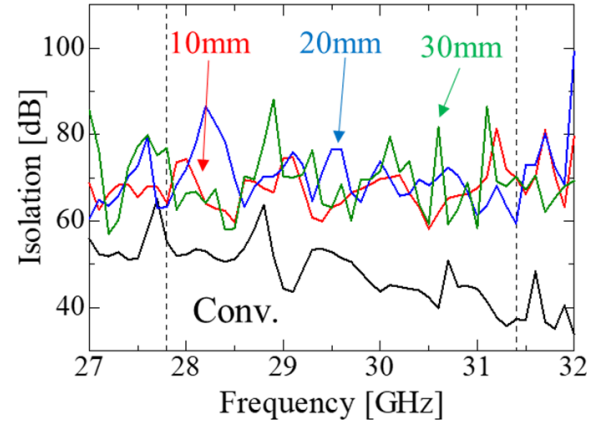

(c) Improvement by only wall

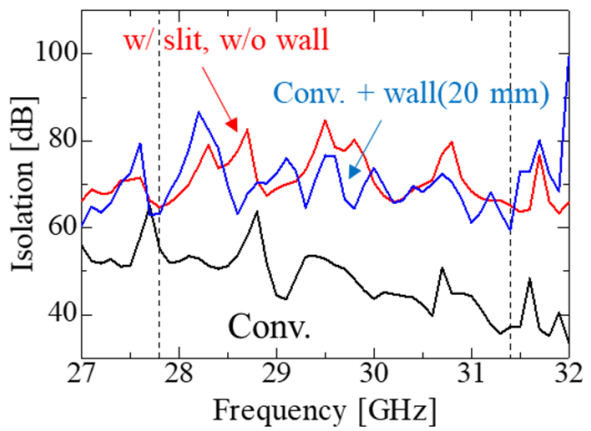

(e) Effect of slit-pair layer and wall

Fig. 2. Model and results of simulation of isolation between $2 \times 32$-element arrays 


\subsection{Improvement by a metal wall between conventional antennas}

Fig. 2(c) shows the improvement by installing a metal wall between two antennas with the conventional structure. The height of the wall is $10 \mathrm{~mm}, 20 \mathrm{~mm}$, and $30 \mathrm{~mm}$. We can observe that about $20 \mathrm{~dB}$ improvement can be realized by using only wall, and there is little difference by the height of the wall.

\subsection{Improvement by a metal wall between proposed antennas}

We install a metal wall between two antennas with the proposed structure. The height of the wall is $10 \mathrm{~mm}, 20 \mathrm{~mm}$, and $30 \mathrm{~mm}$. Fig. 2(d) shows the results. Metal walls cannot give additional improvement to the isolation between proposed antennas without the wall.

\subsection{Improving effect of the proposed structure and a metal wall}

From the above, we can say that the loading oh the slit-pair layer proposed in this manuscript can improve the isolation as much as the installation of a metal wall between the antennas of the conventional structure, as shown in Fig. 2(e). The proposed structure enables us to improve the isolation between E-plane arranged antennas without losing low profile of whole structure.

\section{Measurement at anechoic chamber}

We measure the isolation between E-plane arranged waveguide slot array antennas at

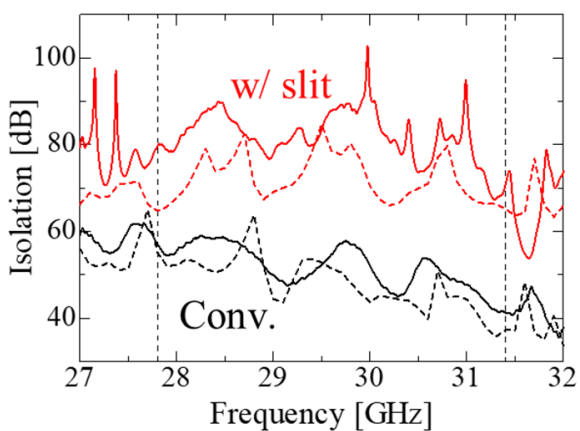

(a) Improvement by slit-pair layer

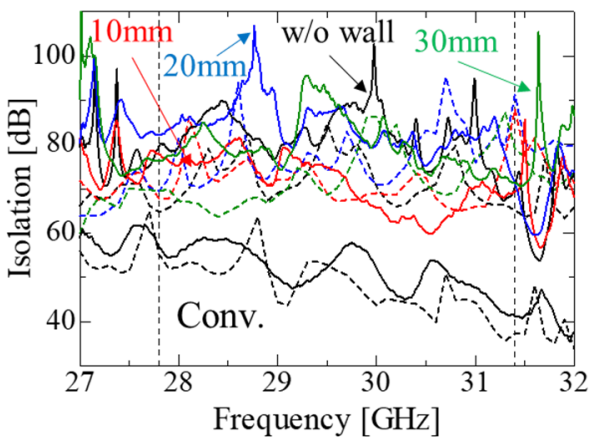

(c) Improvement by slit-pair layer and wall

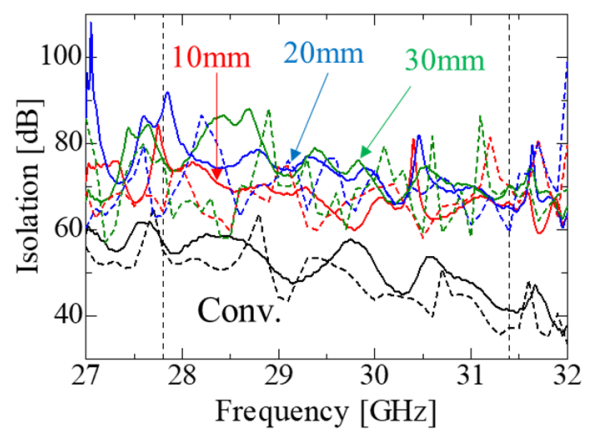

(b) Improvement by only wall

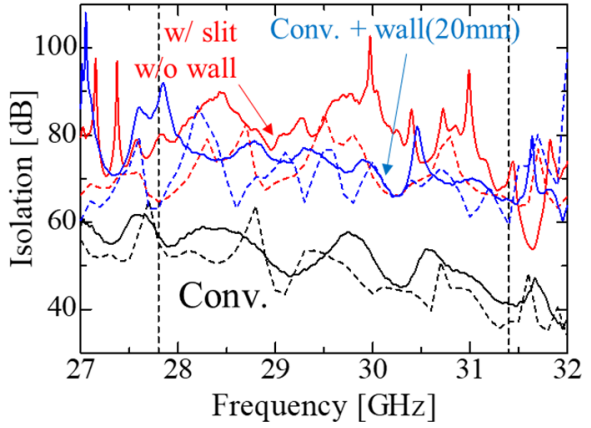

(d) Effect of slit-pair layer and wall

Fig. 3. Comparison of measurement results and simulation results (dashed line: simulation, solid line: measurement) 
an anechoic chamber. A vector network analyzer is used for this measurement, and $64 \times 32$-element arrays are used for transmitting and receiving antenna. Fig. 3(a)-(d) compare the measured results to the simulated one shown in Fig. 2. Broken lines are the simulated results, and solid lines are the measured results.

Each graph shows that the measured results could demonstrate the simulated ones, and the improvement of the proposed structure is comfirmed.

\section{Conclusion}

We have proposed loading layers of slit pairs with a half-wavelength spacing over a double-layer corporate-feed waveguide slot array antenna to improve the isolation between E-plane arranged antennas. We have found that the proposed structure can improve the isolation by about $20 \mathrm{~dB}$ compared to the conventional structure, and the proposed structure has an improvement equivalent to placing a wall between the Tx and $\mathrm{Rx}$ antennas of the conventional structure. We have demonstrated these results by both simulation and measurement. 